\title{
74
}

type par le Conseil. L'une, de toile et bois, à sections de huit lits, se juxtaposant de manière à former une ambulance indéfinie (modèle de M. le Dr Riant); l'autre, de forme ogivale, à ossature de fer, sans piquets ni cordes (système Tollet).

Enfin, dans une dernière séance, l'assemblée \{examina divẹses questions, et émit différents vœux tendant notamment à recommander la création de Comités de dames, à engager les Comités à compléter le cadre de leurs membres correspondants, et à inviter le Conseil à faire, auprès du gouvernement, des démarches propres à provoquer une loi réprimant les abus du signe de la CroixRouge.

\section{LE MUSÉE INTERNATIONAL DE PARIS}

\section{A M. Gustave Moynier, président du Comité international de la Croix-Rouge.}

Paris, 26 mars 1887.

Monsieur le Président,

Le moment me paraît favorable pour vous entretenir d'un sujet qui, depuis vingt ans, n'a cessé d'intéresser vivement les membres des Sociétés de secours aux blessés militaires. Il s'agit des musées internationaux de la Croix-Rouge.

Lor's de l'exposition universelle de 1867, en voyant réunis en grand nombre, sous le drapeau de la Convention de Genève, des objets de matériel sanitaire des armées de terre et de mer de tous les pays, la pensée me vint de fonder un " musée conservatoire international de la Croix-Rouge " à Paris.

Je proposai à la première conférence des Sociétés de secours aux blessés militaires, qui se tenait à Paris, de créer cette institution, afin de permettre, d'une manière permanente, des études comparatives, en vue d'améliorer les conditions de transport et de traitement des blessés et malades des armées en campagne. Cette proposition fit l'objet d'un rapport présenté par M. de Luck, délégué de S. A. R. le prince Charles de Prusse, grand-maitre de l'Ordre de Saint-Jean de Jérusalem.

Le Musée reçut de nombreux dons de tous les pays.

Lorsque la seconde conférence des Sociétés de secours aux 
blessés militaires se tint à Berlin, en 1869 , la résolution suivante fut votée à l'unanimité :

- Il est à désirer que, dans un but d'utilité publique et dans l'in" térêt de la propagation de toutes les inventions qui peuvent - tendre à l'adoucissement du sort des militaires blessés ou ma- lades, il se forme dans chaque pays, ou pour une réunion de - plusieurs pays, en proportion des ressources dont on pourra dis" poser, des collections d'objets de matériel sanitaire exposées d'une " manière permanente. '

Dans la troisième conférence de Genève, en 1884, entre autres résolutions relatives au matériel d'ambulance, il en fut voté une pour décider le renvoi à la prochaine conférence de l'étude de l'organisation de musées internationaux du matériel d'ambulance.

Aujourd'hui, le Musée-conservatoire international de la CroixRouge, fondé à Paris en 1867, est installé à l'Hòtel des Invalides.

Cette création, due à l'initiative privée, conserve son caractère d'indépendance et d'internationalité.

Le Conservatoire s'enrichit chaque jour de toutes les inventions et de tous les perfectionnements concernant le matériel sanitaire des armées.

Jl est destiné à être un centre d'études et d'expériences comparatives.

En même temps qu'ils s'efforceront de recueillir dans tous les pays des documents et des objets utiles à ses collections, ses administrateurs propageront ce qu'ils auront jugé bon de faire connaître dans l'intérêt de l'humanité.

Le Comité d'administration du musée se compose de délégués de plusieurs gouvernements. Les gouvernements d'Allemagne, d'Angleterre, d'Autriche-Hongrie, d'Italie, de Portugal, de Russie, de Suède el Norvège, ont choisi pour cela leurs attachés militaires.

Les ministres de la guerre et de la marine de France ont nommé également des délégués.

Les dépenses de cet établissement ont été couvertes jusqu'ici, et continueront à l'être, à l'aide de dons particuliers. Les délégués, non plus que leurs gouvernements, n'ont à contribuer, en quoi que ce soit, aux frais du musée.

J'ai l'espoir, Monsieur le Président, que, lors de nos prochaines conférences à Carlsruhe, les membres de la Croix-Rouge voudront bien reconnaîlre que nos efforts incessants, depuis vingt ans, n'ont 
pas été sans quelque utilité, pour l'étude des améliorations du matériel sanitaire des armées et les sérieux progrès qui ont été obtenus.

Veuillez croire, Monsieur le Président, à mes sentiments dévoués.

Comte Sḱnurier.

\section{COMITÉ DÉPARTEMENTAL DE L'ISĖRE}

Le personnel des infirmiers du Comité départemental de l'Isère a exécuté en 1886, à deux reprises différentes, des manœuvres d'ambulance. Il s'agissait de savoir si les fourgons pourraient être facilement conduits dans la monlagne, et quelles seraient les difficultés qui s'y rencontreraient. Une expédition a été faite à cet effet dans les environs de Grenoble. Une maison avait été transformée en ambulance; les brancardiers ont fait des manœuvres pour relever des blessés dans des parages accidentés, tels que des éboulis, et pour transporter des blessés dans des passages difficiles.

Les exercices mensuels des brancardiers ont eu pour but, pendant l'année 1886, d'étudier les moyens de transformer des wagons de marchandises en wagons sanitaires. Aux mois de décembre et de janvier, des exercices pour l'embarquement et le débarquement des blessés ont eu lieu à la gare des marchandises à Grenoble.

L'activité déployée par le Comité des dames a pourvu la lingerie d'un stock considérable de linge confectionné pour pansements. Grâce au concours du Comité de Paris, le Comité de Grenoble a acquis une série de substances antiseptiques, qui lui permettront de préparer en quelques jours plusieurs milliers de pansements. D'un-autre côté, une réserve de médicaments jarcotiques Iui permeltra de secourir plus de cinq mille blessés ou malades. Enfin, le Comité, toujours préoccupé de l'idée d'être en mesure de faire face à tous les événements, continue à augmenter chaque année son matériel; il a acquis, en 1886, deux pharmacies portatives, 50 brancards, deux tables d'opérations et une boîte de chirurgie pourvue des instruments les plus perfectionnés. 